\title{
Subcellular fraction associated to radionuclide analysis in various tissues: Validation of the technique by using light and electron observations applied on bivalves and uranium
}

\author{
O. Simon, V. Camilleri, G. Grasset and J. Garnier-Laplace \\ Laboratory of Radioecology and Ecotoxicology, Institute for Radiological Protection and \\ Nuclear Safety, DEI/SECRE/LRE, Cadarache, Bât. 186, BP. 3, 13115 St-Paul-lez-Durance \\ Cedex, France
}

\begin{abstract}
The metal bioaccumulation levels in target-organs associated with microlocalisation approaches at the subcellular level provide information for the understanding of the metabolic metal cycle. These findings could be used to select relevant biomarkers of exposure and to focus on specific contaminated organelles to study potential biological effects. Moreover, the metal accumulated in the cytosol fraction can be bound to macromolecules in order to be eliminated and/or to induce a potential cellular effect. Tissular distribution, transfer efficiency from water and subcellular fractionation were investigated on the freshwater bivalve, Corbicula fluminea after uranium aqueous exposure. The subcellular fractionation was performed while measuring associated uranium to each cellular different fraction as follows: cellular debris and nuclei, mitochondria and lysosomes, membranes, microsomes and cytosol. In our experimental conditions, the accumulation in the cytosol fraction was low and more than $80 \%$ of the total uranium in gills and visceral mass was accumulated in the insoluble fraction. Main results presented in this paper come from light and electron microscope observations of subcellular fractions (nuclei/debris and lysosomes/mitochondria) in order to validate the efficiency of the fractionation technique. An adaptation of the fractionation technique is proposed. This set of data confirms high differences of fractionation efficiency as a function of used fractionation technique and organs/biological model.
\end{abstract}

\section{INTRODUCTION}

The study of the impact of pollutant exposure on living organisms gathers at least three research fields; (i) the link between pollutant in the ecosystem and its efficiency to be accumulated, (ii) the internalization (bio)markers and (iii) the potential involved effects on organisms. The link between exposure, internalization and toxic effects must be investigated for both exposure routes, the direct contamination from the concentration of pollutant in water and the trophic contamination from contaminated prey ingestion [1]. This paper is focused on the internalization markers such as the accumulation levels of the pollutant and its organs distribution. These markers could be used to reflect pollutant exposure and allow to focus on target-organs. For example, it is well known that the gills of a freshwater organism are the target organ of the direct exposure and consequently a part of research is focused on this organ [2]. To complete this information, the microlocalisation of pollutant in main organelles are required to understand the mechanism by which aquatic animals respond to pollutant exposure. For many metals, the accumulation in the cytosol fraction of the cell indicates a link between functional macromolecules and metal, and can be correlated to the toxic effects. For example, the cadmium aptitude to induce the metallothionein synthesis, accumulation in the cytosolic protein fractions could explain the high concentration levels measured in bivalves. The most widely used 
technique for fractionating cellular components is the use of a differential centrifugation technique to separate into four main subcellular fractions (Nuclei and cellular debris, mitochondria and lysosomes, microsome, membranes) and the cytosolic fraction. Note that guidelines for cell fractionation indicate that this technique does not lead to pure fraction, but a number of authors [3-5] use such a technique. Finally, references indicated several techniques to obtain subcellular fractions; the main differences were based on the tissue homogenisation procedure and the use of filtration after the homogenization step. Despite the wide variation in freshwater uranium concentration from $5.2 \mu \mathrm{g} . \mathrm{L}^{-1}$ to $2 \mathrm{mg} . \mathrm{L}^{-1}$ [6], little information is currently available on its impact on ecosystems [7]. The first step of the approach consisted in measuring the uranium capacity to be accumulated and the $U$ organs and cellular distribution. Due to its low specific activity, natural uranium in freshwater ecosystem is very often considered to be a significant chemical hazard $[8,9]$. Taking into account the high capacity of metal accumulation and its use in ecotoxicology [4], the bivalve Corbicula fluminea was chosen to measure the $\mathrm{U}$ accumulation capacity. The main objective of this preliminary study was to determine the target-organ of natural uranium exposure via the water column at a quite high level of exposure (500 $\mu$ g. $\mathrm{L}^{-1}$, during 10 days of exposure), to increase the chance to identify the main subcellular fraction of the $U$ accumulation. Complementary experiments were performed to measure the impact of two subcellular fractionation techniques in order to validate the $\mathrm{U}$ cellular target, by using light and electronic observations of subcellular fractions.

\section{MATERIALS AND METHODS}

\subsection{Collection of bivalves}

The freshwater bivalves, $C$. fluminea (soft body mass $=1.65 \pm 0.29 \mathrm{~g}$ fresh weight (fw), $\mathrm{n}=40$ ) were manually collected from a site on the bank of the Moselle river (Moselle, France). The background uranium level was below the analytical detection limit $\left(50 \mathrm{ng} \cdot \mathrm{g}^{-1}, \mathrm{fw}\right.$ ) of the ICP-AES equipment. Bivalves were maintained in a storage tank (sand + water) and were fed an algae suspension twice a week. The ionic composition of artificial water was identical during acclimatisation and exposure phases.

\subsection{Direct exposure conditions and tissue analysis and subcellular fractionation procedure}

Two exposure treatments were performed; a U exposure condition ([U $]_{\text {in water }}=500 \mu \mathrm{g} / \mathrm{L}$ ) and a control (without $\mathrm{U}$ ) condition, over 10 days. The experimental system per condition consisted of two connected tanks. The first held $300 \mathrm{~L}$ of synthetic water at constant $\mathrm{pH}(\mathrm{pH}$ regulation Consort R305) and with a constant uranium level in water. The second tank, named the experimental unit contained $25 \mathrm{~kg}$ of sand and $50 \mathrm{~L}$ of aerated synthetic water (by bubbling air) and bivalves ( 40 individuals). The flow rate was selected to ensure the complete renewal of the experimental unit within $24 \mathrm{~h}$. The water concentrations in both tanks were measured by ICP AES (water sample was acidified at 2\%) twice a day and the loss of U was compensated by daily manual additions. At the end of 10 days of exposure, gills and visceral mass were sampled. The dissection of bivalves $(\mathrm{n}=6)$ was performed on living organisms to allow the light and electronic observations and the subcellular fractionation. A piece of organ was used to measure the $\mathrm{U}$ accumulation levels at the organs levels. After dissection, each organ sample was stored in freezer and then was mineralised in a propylene tube using nitric acid ( $3 \mathrm{ml}, 65 \%$ ) and perchloric acid ( $2 \mathrm{ml}, 33 \%)$ at $105^{\circ} \mathrm{C}$ for $3 \mathrm{~h}$. Samples were then diluted, acidified (2\%) and spiked with a well-known quantity of yttrium for ICP-AES analysis. The quality control sample was prepared by addition of a known concentration of uranium to a mineralised $C$. fluminea tissue. The second part of both organs was used to obtain subcellular fractions. Two techniques of homogenisation were tested. As tissue-grounder, the first technique used an ultra-thurrax although the second used a Potter-Elverhjem glass homogeniser. Moreover, for technique 2, the homogenate was filtrated (F, $10 \mu \mathrm{m}$ ) before centrifugations. After homogenisation, the centrifugation technique was identical for both techniques. The subcellular fractionation involved four 
successive differential centrifugations (Beckman Coulter, rotor JS 24 15, USA) in order to obtain four types of cellular structures (at $4^{\circ} \mathrm{C}, \mathrm{C} 1$ : nuclei, cellular debris and granules: $900 \mathrm{~g}$ for $10 \mathrm{mn}$; C2: mitochondria and lysosomes: $12000 \mathrm{~g}$ for $15 \mathrm{mn}$; C3: membranes: 45 000g for $30 \mathrm{mn}$; C4: microsomes: $103000 \mathrm{~g}$ for $70 \mathrm{mn}$ ) and the cytosol (S) fraction) [10]. The four pellets and the cytosol fraction have followed the U analysis described previously. The subcellular distribution is expressed in percentage between each $U$ cellular fraction and the $U$ amount for all the fractions. The light and electronic observations of the first and second pellets were performed from other exposed bivalves.

\subsection{Light and electronic observations}

The first two pellets were routinely fixed with $1.5 \%$ glutaraldehyde in sodium cacodylate buffer $(\mathrm{pH} 7.4)$ during 24 hours at $4^{\circ} \mathrm{C}$. They were then washed in sodium cacodylate buffer and post-fixed in the same buffer containing $1 \%$ OsO4. Dehydration took place in a grades series of alcohols and Epon was used as the embedding medium. Semithin sections $(1.5 \mu \mathrm{m})$ stained with aqueous blue toluidine were observed under a light microscope (Nikon, Eclipse E 400) and images were saved with a Sony camera and a Biocom Visiol Software. Ultrathins $(110 \mathrm{~nm})$ were observed with a Scanning Transmission Electron Microscope (TEM/STEM, Tecnai, FEI company), equipped with CCD camera and Energy-Dispersive-X-Ray (EDAX) analysis.

\subsection{Statistical analysis}

Mean and standard deviation from 6 replicates per condition were calculated for all bioaccumulation parameters. The impact of the used techniques on the accumulation levels in each subcellular fraction was checked by using a student's t test $(\mathrm{p}=0.05$, Sigma Stat software).

\section{RESULTS AND DISCUSION}

\subsection{U accumulation levels in gills and visceral mass}

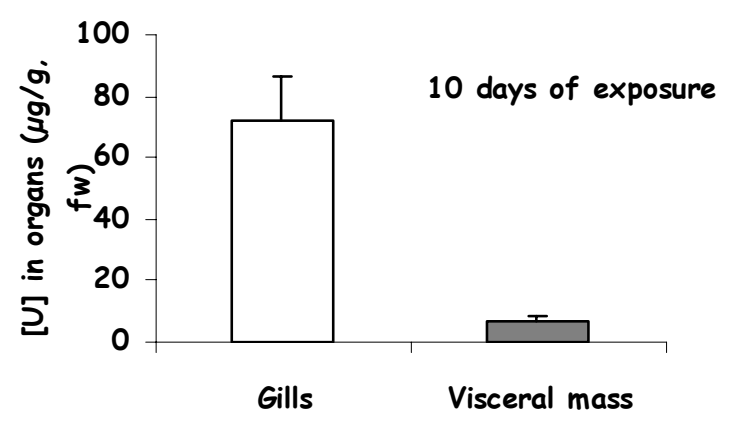

Figure 1. Accumulation ( $\left.\mu \mathrm{g} . \mathrm{g}^{-1}, \mathrm{fw}\right)$ in gills and visceral mass of the $\mathrm{U}$ exposed bivalve $C$. fluminea after 10 days of exposure (mean $\pm \mathrm{SD}, \mathrm{n}=6$ ).

The average concentration in water measured daily was close to the nominal concentration. The accumulation levels $\left(\mu \mathrm{g} . \mathrm{g}^{-1}, \mathrm{fw}\right)$ in gills and visceral mass of $\mathrm{U}$ exposed bivalves are shown in Figure 1. The gills accumulation $\left(72 \pm 6.5 \mu \mathrm{g} \cdot \mathrm{g}^{-1}\right)$ was higher (x5) than the accumulation level in the visceral mass $\left(14.4 \pm 2 \mu \mathrm{g} \cdot \mathrm{g}^{-1}\right)$. In our experimental conditions, gills are the target-organs of the direct exposure route. 
Such results have been described for many freshwater organisms and heavy metals [2,11]. Note that previous results have indicated that the level of water exposure influences the role of the gills. For low level of $U$ exposure, the higher accumulation level was measured in the visceral mass although for high level of exposure (500 $\left.\mu \mathrm{g} . \mathrm{L}^{-1}\right)$, the gills seemed to be the main target [12]. Without $U$ contaminated food or particles, in our experimental conditions, the visceral mass accumulation level is not due to the trophic exposure route. The visceral mass could be involved in the $U$ sequestration in a process of detoxification mechanism from a $\mathrm{U}$ gills flux. The $\mathrm{U}$ efficiency of the water/organism transfer could be evaluated by

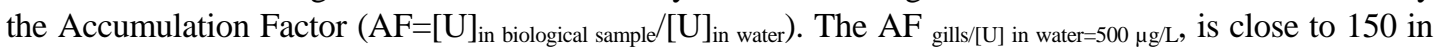
these experimental conditions. Such a value in gills of $C$. fluminea has been measured for another exposure condition for which the differences mainly concerned the $\mathrm{pH}$ value of 7 . Consequently, the free ion $\mathrm{UO}_{2}{ }^{2+}$ for which the distribution in water is strongly influenced by the $\mathrm{pH}$ value (contribution to the total $\mathrm{U}$ concentration: $<10^{-2} \%$ at $\mathrm{pH} 7 ; 1 \%$ at $\mathrm{pH} 6.5$ ) seems not to play a significant role in the gills accumulation. Other uranium dissolved species could contribute to the $\mathrm{U}$ accumulation within bivalves. These results show that the uranium is transferred from the water to the organism and the potential effect of $U$ accumulation could appear in the gills.

\subsection{Comparison of accumulation levels in each subcellular fractions}

\subsubsection{U subcellular distribution}

The following step to assess the $\mathrm{U}$ internalisation markers concerns the $\mathrm{U}$ subcellular distribution in the main organs of bivalve; the gills and the visceral mass. Figure 2 shows the accumulation levels in each of the 4 subcellular fractions, in cytosol fraction (S) and in filtrate (F), both organs as a function of used techniques. The comparison of both techniques allows us to discuss the efficiency of the "homogenisation+centrifugation" technique.

\section{Uranium distribution (\%) in each subcellular fraction}

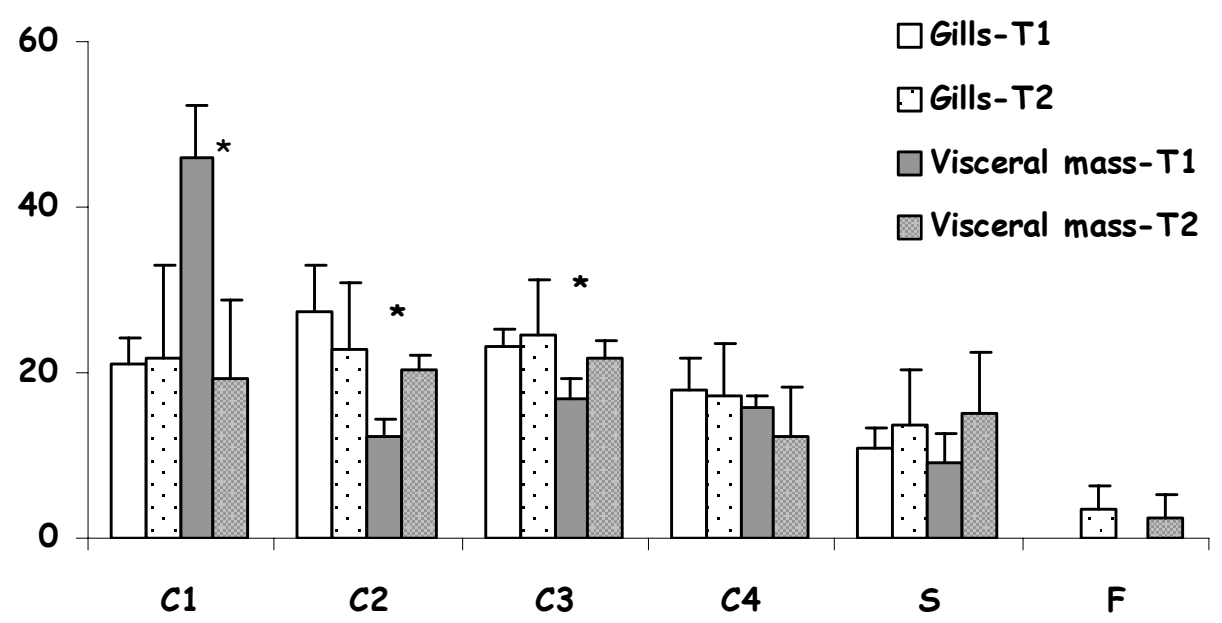

Figure 2. U distribution (\%) in 4 subcellular fractions of gills and visceral mass of the $U$ exposed bivalve C. fluminea after 10 days of exposure as a function of the used technique.* index statically difference $(\mathrm{p}<0.05)$ between technique 1 and technique $2 \mathrm{U}$ distribution. C1: nuclei, cellular debris and granules, C2: mitochondria and lysosomes, C3: membranes, C4: microsomes, S: cytosol,F: filtrate. 
Significant differences are highlighted between C1, C2 and C3 fractions of the visceral mass between the two techniques. Technique 1 leads to the U distribution in C1: $45 \pm 6 \%$, C2: $12 \pm 2 \%$ and C3: $16 \pm 2.5 \%$ fractions although technique 2 leads to C1: $24 \pm 6 \%$, C2: $19 \pm 2 \%$ and C3: $22 \pm 2 \%$. For the visceral mass condition, the amount of the three first pellets for technique 1 and the three pellets + filtrate for technique 2 is identical. For the gills subcellular fractions, the $U$ distribution is identical between the 4 pellets, close to $20 \%$. No significant difference is observed between the two techniques. For both organs, the quantity of $U$ in the filtrate is low in spite of the high mass of biological material in the filtrate. Figure 2 shows a significant difference of $U$ distribution in the subcellular fractions of the visceral mass although no difference is observed for the gills. Results suggest that the efficiency of the homogenization modifies the results of the U distribution in subcellular fractions. In this case, it is difficult to use these results to discuss the U subcellular distribution.

\title{
3.2.2 Efficiency of the subcellular fractionation by using light and electronic observations
}

The light and electronic observations of the first two pellets are presented on figure 3. These photographs tend to sum up the amount of observations. The light observations were performed from the first pellet of each technique. The electronic observations were focused on the first (C1) and second pellets (C2) of the gills from technique 2 .
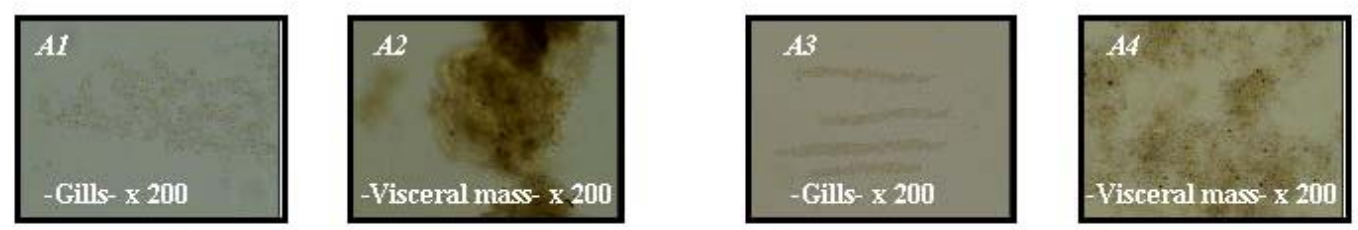

C1: Nuclei and cellular debris C2: Mitochondria and lysosoms
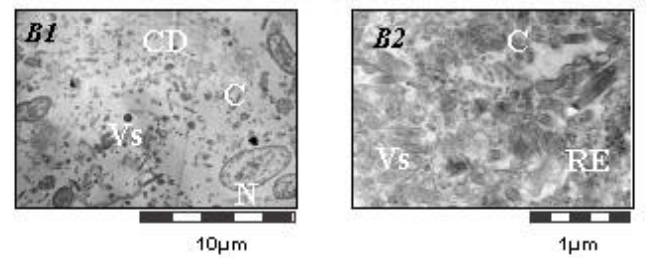

\author{
C: Cilia \\ CD: Cellular debris \\ N: nuclei \\ RE: Endoplasmic reticulum \\ Vs: vesicules
}

Figure 3. A. Light observations of the first pellets of the gills and visceral mass as a function of used technique 1(A1-A2) \& 2 (A3-A4). B. Electronic observations of the two pellets (Technique 2) of the gills.

The visceral mass homogenisation seems to be more efficient with a Potter as tissue-grounder (A4) but leads to a high rate of non-homogenised tissues for which the successive centrifugations did not allow to correctly separate the subcellular fractions. Consequently, following the technique 1 separation, the first pellet contains a high burden of non-homogenised tissue and could explain the high $U$ distribution in the C1 pellet. The better homogenisation of visceral mass (Technique 2) could explain the $U$ distribution in the three pellets. Note that in both cases, a high proportion of tissue was not homogenised. The light observations confirm that the gills homogenisation (A1-A3) with an ultra-thurrax or a Potter is comparable. The electronic observations were performed on the gills, organ for which the light observation did not highlight a significant difference of the homogenisation efficiency. The gills electronic observations show that the first two centrifugations do not allow to obtain weight and length separations, little vesicles and cilia of the gills epithelium (potential sites of $U$ accumulation) are found in $\mathrm{C} 1$ and $\mathrm{C} 2$ fractions. Consequently, $\mathrm{U}$ accumulation levels in both fractions are not representative of the $U$ burden in nuclei and cellular debris and mitochondria and lysosome respectively. Finally, the information in terms of accumulation level in the cytosol fraction must be 
taken with precaution despite the theoretically effective separation; the supernatant of the last centrifugation (103 $000 \mathrm{~g}$ ) contains only dissolved elements. Indeed, each U cellular fraction is expressed in percentage of the all fractions. The $U$ cytosol fraction depends on the total $U$ burden measured in all pellets. The ineffective homogenisation of the visceral mass leads to the presence of tissue in the first pellets as described by light observations. A part of the cytosol will be found in the pellet. The $U$ measured in the cytosol fraction collected in the supernatant of the last centrifugation does not take this part into account. The \% of $U$ in the cytosol depends directly on the efficiency of the homogenisation according to the other pellets. Consequently, the $U$ cellular separation is not acute enough to allow secondary the $U$ analysis in each subcellular fraction. In our experimental conditions, it seems to be wrong to compare the $U$ distribution in the 4 pellets and in cytosol fraction between the two organs without knowing the efficiency of cytosol extraction from each tissue. By using this technique, no significant subcellular target can be highlighted in order to complete ecotoxicological informations.

\section{CONCLUSION}

The article presents preliminary results of the $U$ accumulation in bivalve after high exposure level in the water. In our exposure conditions, the gills are the target-organs of direct exposure route. Associated to other results, we demonstrated that the accumulation level in gills are not directly linked to the free ion $\mathrm{UO}_{2}{ }^{2+}$ and that other dissolved uranium species can be responsible for the $\mathrm{U}$ accumulation. The main results are focused on the comparison of two techniques of tissue homogenisation before successive and differential centrifugations. The accumulation levels and the light/electronic observations show a significant effect of the used homogeniser and demonstrate that the homogenisation efficiency depends on the organs. Consequently, this technique cannot be used to compare the U cellular distribution between organs and organisms. Other biochemical techniques will perform to obtain an effective tissue homogenisation (sonication phase after tissue-grounder/ preliminary step before tissue homogeneisation: Cell dissociation by trypsin/collogenase).

Finally, the microlocalization of $U$ at the cellular level will be performed with the transmission electron microscope equipped with Energy-Dispersive-X-Ray (EDAX) analysis in order to identify the organelles of the $\mathrm{U}$ accumulation.

\section{References}

[1] Fisher N.S, Hook S.E,. Toxicology 181-182 (2002) 531-536.

[2] Dallinger R., Prosi F., Segner H., Back H., Oecologia 73 (1987) 91-98.

[3] Romeo M., Gnassia-Barelli M., Comp. Biochem. Physiol. 111 (1995) 457-463.

[4] Vesk P.A., Byrne M., Sci. Total Environ. 225 (1999) 219-229.

[5] Wallace W.G., Lopez G.R., Estuaries 19 (1996) 923-930.

[6] Betcher R.N., Gascoyne M., Brown D., Can. J. Earth. Sci. 25 (1998) 2089-2103.

[7] Colle C., Garnier-Laplace J., Roussel-Debet S., Adam C., Baudin J.P., L'uranium de l'environnement à l'homme (EDP Sciences, Les Ulis, 2001) pp. 187-211.

[8] Cooley H.M., Evans R.E., Klaverklamp J.F., Aquatic Toxicology 48 (2000) 477-494.

[9] Khune W.W., Caldwell C.A., Gould W.R., Fresquez P.R., Finger S., Envirom. Toxicol. Chem. 21 (2002) 2198-2203.

[10] Paquet F., Etude expérimentale des biocinétiques de l'americium-241 chez le homard Hommarus gammarus. Analyse des mécanismes d'accumulation et de détoxication au niveau subcellulaire (Rapport CEA-R-5629, 1993) pp58-61.

[11] Marigomez I., Baybay-Villacorta L., Aquat. Toxicol. 64 (2003) 235-257.

[12] Simon O., Garnier-Laplace J., Aquatic Toxicology 68 (2004) 95-108. 